\title{
Originales
}

\section{Saturación arterial de oxígeno durante la ascensión a una montaña de más de 8.000 metros}

\author{
J. BOTELLA DE MAGLIA ${ }^{a, b, c}$, R. REAL SORIANO ${ }^{b, c}$ Y L. COMPTE TORRERO ${ }^{d}$ \\ aUnidad de Medicina Intensiva. Hospital La Fe. Valencia. España. \\ ${ }^{\text {b} E x p e d i c i o ́ n ~ C i n c ~ S e g l e s ~ d e ~ l a ~ U n i v e r s i t a t ~ d e ~ V a l e ̀ n c i a ~ a l ~ G a s h e r b r u m ~ I I . ~ E s p a n ̃ a . ~}$ \\ 'Sociedad Española de Medicina y Auxilio en Montaña (SEMAM). España. \\ dServicio de Neumología. Hospital La Fe. Valencia. España.
}

Fundamento. La saturación arterial de oxígeno $\left(\mathrm{SaO}_{2}\right)$ disminuye con la altitud.

Objetivo. Conocer qué $\mathrm{SaO}_{2}$ presentan los montañeros sanos durante la ascensión a una montaña de más de 8.000 metros.

Método. En una expedición al Gasherbrum II $(8.035 \mathrm{~m})$ se midió la $\mathrm{SaO}_{2}$ por pulsioximetría durante la marcha de aproximación, en el campamento base (a la llegada y un mes después), en los campamentos II y III, a 7.500 m durante el ataque y en la cumbre.

Resultados. En la marcha de aproximación la $\mathrm{SaO}_{2}$ en Paiyu (3.365 m) fue del $92,9 \pm 1,4 \%$ y en Gore II $(4.250 \mathrm{~m})$ del 85,0 \pm 4,3\%. En el campamento base $(5.200 \mathrm{~m})$ a la llegada fue del 78,4 \pm $9,5 \%$ y un mes después del $87,4 \pm 3,0 \%(p<0,007)$. En el campamento II $(6.500 \mathrm{~m})$ fue del $72,7 \pm 6,7 \%$. En el campamento III $(7.000 \mathrm{~m})$ fue del $68,0 \pm 9,3 \%$ (medida sobre 21 sujetos). A esta altitud se registró una $\mathrm{SaO}_{2}$ del $40 \%$ durante el sueño en un sujeto asintomático. A $7.500 \mathrm{~m}$ fue del 60,5 \pm $13,5 \%$ (4 sujetos). En la cumbre $\left(8.035 \mathrm{~m}\right.$ ) la $\mathrm{SaO}_{2}$ de dos sujetos fue del 84 y $88 \%$ respectivamente.

Conclusión. Durante las expediciones a montañas de más de $\mathbf{8 . 0 0 0} \mathrm{m}$ los montañeros presentan cifras de $\mathrm{SaO}_{2}$ muy bajas, comparables a las de los pacientes con insuficiencia respiratoria grave. La $\mathrm{SaO}_{2}$ aumenta progresivamente con la

Correspondencia: Dr. J. Botella de Maglia.

C/ Císcar, 25.

46005 Valencia. España.

Manuscrito aceptado el 8-I-2008. aclimatación. Es posible que la $\mathrm{SaO}_{2}$ en la cima del Gasherbrum II fuera relativamente alta, probablemente a consecuencia de la hiperventilación.

PALABRAS CLAVE: saturación arterial de oxígeno, alta montaña, montañismo.

\section{ARTERIAL OXYGEN SATURATION DURING ASCENT OF A MOUNTAIN HIGHER THAN 8,000 METERS}

Background. Arterial oxygen saturation $\left(\mathrm{SaO}_{2}\right)$ diminishes with altitude.

Aim. To know the values of $\mathrm{SaO}_{2}$ in healthy mountaineers during the ascent of a mountain higher than 8,000 metres.

Method. On occasion of an expedition to Gasherbrum II $(8,035 \mathrm{~m}), \mathrm{SaO}_{2}$ at rest was measured by pulse oxymetry during the approach march, in the base camp (on day one and one month later), in camps II and III, during the assault at $7,500 \mathrm{~m}$ and on the summit.

Results. During the approach march, the $\mathrm{SaO}_{2}$ in Paiju $(3,365 \mathrm{~m})$ was $92.9 \pm 1.4 \%$ and in Gore II $(4,250 \mathrm{~m}) 85.0 \pm 4.3 \%$. In the base camp $(5,200 \mathrm{~m})$ it was $78.4 \pm 9.5 \%$ on the first day and $87.4 \pm 3.0 \%$ one month later $(p<0.007)$. In camp II $(6,500 \mathrm{~m})$ it was $72.7 \pm 6.7 \%$. In camp III $(7,000 \mathrm{~m})$ it was 68.0 $\pm 9.3 \%$ (recorded on 21 asymptomatic climbers). At this altitude a $\mathrm{SaO}_{2}$ of $40 \%$ was recorded during sleep in an asymptomatic subject, apparently without apnoeic crises. During the assault at $7,500 \mathrm{~m}, \mathrm{SaO}_{2}$ was $60.5 \pm 13.5 \%$ (measured on 4 climbers). On the summit $(8,035 \mathrm{~m})$ the $\mathrm{SaO}_{2}$ of two subjects was $84 \%$ and $88 \%$, respectively.

Conclusion. During expeditions to mountains higher than 8,000 metres, mountaineers have ex- 
tremely low values of $\mathrm{SaO}_{2}$, similar to those of patients with severe respiratory failure. $\mathrm{SaO}_{2}$ increases progressively with acclimatization. $\mathrm{SaO}_{2}$ on the summit could have been relatively high, probably because of hyperventilation.

KEY WORDS: arterial oxygen saturation, high altitude, mountaineering.

\section{INTRODUCCIÓN}

La saturación arterial de oxígeno $\left(\mathrm{SaO}_{2}\right)$ disminuye con la altitud. En un estudio anterior hemos puesto de manifiesto que, para una altitud dada, $\mathrm{la} \mathrm{SaO}_{2}$ de las personas que residen habitualmente a esa altitud es mayor que la de los montañeros no aclimatados. Como resultado de nuestras observaciones hemos elaborado ecuaciones que nos permiten determinar, para cualquier altitud por debajo de $4.200 \mathrm{~m}$, qué cifras de $\mathrm{SaO}_{2}$ podemos considerar normales para los montañeros no aclimatados y para los habitantes de las montañas ${ }^{1}$. Sin embargo, nuestras ecuaciones no son aplicables a la altitud extrema.

La altitud extrema (>5.500 m) es aquella en la que la hipoxia impide la vida humana permanente. No existen en ella poblaciones humanas estables, ni cabe hablar de montañeros no aclimatados. Las montañas de más de $5.500 \mathrm{~m}$ suelen estar ubicadas en cordilleras remotas que requieren largas marchas de aproximación durante las cuales los montañeros se aclimatan parcialmente. Las ascensiones se realizan siempre en condiciones de aclimatación parcial. No se pueden hacer sin aclimatación porque el montañero sufriría mal de montaña, y podría morir por un edema cerebral o un edema pulmonar de la altitud, pero tampoco se pueden hacer con una aclimatación completa porque para alcanzarla habría que permanecer mucho tiempo en altitudes extremas y el montañero acabaría debilitado por el deterioro muscular de la altitud.

La expedición Cinc Segles de la Universitat de València al Gasherbrum II nos dio la oportunidad de estudiar cuáles son las cifras de $\mathrm{SaO}_{2}$ que tienen los montañeros durante la ascensión a una montaña de más de $8.000 \mathrm{~m}$. A diferencia de nuestros estudios anteriores, en esta ocasión no se trataba de comparar la $\mathrm{SaO}_{2}$ de los montañeros no aclimatados con la de los habitantes de las montañas, sino de medir la $\mathrm{SaO}_{2}$ en las circunstancias alpinísticas reales que se dan en este tipo de expediciones, independientemente del mejor o peor estado de aclimatación.

\section{SUJETOS Y MÉTODO}

El Gasherbrum II es una montaña de 8.035 m ubicada en el Karakorum. La expedición Cinc Segles de la Universitat de València, que tuvo lugar en el verano de 1999, fue uno de los actos con los que se conmemoró el 500 aniversario de la fundación de la Universidad de Valencia. Su desarrollo se describe pormenorizadamente en otro lugar² ${ }^{2}$. Además del estudio que nos ocupa, durante la expedición se realizaron también otras investigaciones de fisiología respiratoria, cuyos resultados ya se han dado a conocer ${ }^{3}$.

En esta expedición participaron una mujer y 7 hombres, todos ellos montañeros sanos, de edades comprendidas entre los 32 y 42 años $(38,2 \pm 3,4$ años). Todas las mediciones a las que se refiere este estudio se efectuaron sobre estos sujetos, a excepción de las realizadas en el campamento III, en el que se hizo pulsioximetría también a otros 13 sujetos, miembros de una expedición francesa y otra norteamericana.

$\mathrm{La} \mathrm{SaO}_{2}$ se midió por medio de un pulsioxímetro Pulsiox-3 (Minolta, Osaka, Japón), cuya ligereza y pequeño tamaño permitieron que una autora de este estudio (RRS) lo llevase en su muñeca hasta la cumbre como si se tratase de un reloj. Este pulsioxímetro permite el almacenamiento de la información por medio de un sistema tipo Holter.

Todas las mediciones se hicieron dentro de una tienda de campaña y en reposo (como mínimo 5-10 minutos tras el ejercicio), a excepción de la que se realizó en la cima del Gasherbrum II, que se llevó a cabo a la intemperie y tras un periodo de reposo más breve.

$\mathrm{La} \mathrm{SaO}_{2}$ se midió durante la marcha de aproximación, en el campamento base al principio y al final de la estancia en él, en los campamentos II, III y IV, y en la cumbre del Gasherbrum II, según se detalla a continuación:

1. Durante la marcha de aproximación se midió la $\mathrm{SaO}_{2}$ de los 8 expedicionarios el día de descanso que la expedición pasó en el bosque de Paiyu $(3.365 \mathrm{~m})$, antes de entrar en el glaciar Baltoro.

2. Dos días después se midió de nuevo la $\mathrm{SaO}_{2}$ al llegar al lugar llamado Gore II $(4.250 \mathrm{~m})$ sobre el glaciar Baltoro.

3. En el campamento base $(5.200 \mathrm{~m})$ se midió la $\mathrm{SaO}_{2}$ a los 8 expedicionarios al día siguiente a su llegada ( 9 de julio) y un mes después ( 9 de agosto).

4. En el campamento II ( $\sim 6.500 \mathrm{~m})$ se hizo pulsioximetría en dos ocasiones, separadas por un intervalo de 10 días. En la primera, el 26 de julio, a 6 sujetos (una mujer y 5 hombres) de edades comprendidas entre 32 y 42 años. En la segunda, el 5 de agosto, a 4 sujetos (todos varones) entre 32 y 42 años.

5. En el campamento III $(\sim 7.000 \mathrm{~m})$ se hizo pulsioximetría a 21 sujetos (tres mujeres y 18 hombres), de edades comprendidas entre los 25 y 47 años. Esta medición se hizo en reposo, la tarde previa a sus respectivos intentos a cima. En un varón de 42 años se hizo también un registro continuo de la $\mathrm{SaO}_{2}$ durante la noche previa al ataque.

6. Durante el segundo ataque a la cumbre se hizo pulsioximetría a 4 personas, de edades comprendidas entre los 32 y los 42 años, en el interior de una tienda de campaña abandonada en el campamento IV (7.500 m).

7. En la cima del Gasherbrum II ( $8.035 \mathrm{~m})$ se hizo pulsioximetría a los dos sujetos que la alcanzaron (una mujer de 36 años y un hombre de 42).

Los resultados se expresan como media aritmética \pm desviación típica. La comparación entre la $\mathrm{SaO}_{2}$ 
TABLA 1. Saturación arterial de oxígeno durante la ascensión a una montaña de más de $8.000 \mathrm{~m}$

\begin{tabular}{|c|c|c|c|c|}
\hline Lugar & Altitud (m) & $\mathrm{n}$ & $\mathrm{SaO}_{2}$ & \\
\hline Paiyu & 3.365 & 8 & $92,9 \pm 1,4 \%$ & \\
\hline Gore II & 4.250 & 8 & $85,0 \pm 4,3$ & \\
\hline Campamento base (primer día) & 5.200 & 8 & $78,4 \pm 9,5$ & $\mathrm{p}=0,007$ \\
\hline Campamento base (día 30) & & 8 & $87,4 \pm 3,0$ & \\
\hline Campamento II (primera medición) & $\sim 6.500$ & 6 & $72,2 \pm 6,7$ & \\
\hline Campamento II (segunda medición 10 días después) & & 4 & $75,5 \pm 1,7$ & \\
\hline Campamento III & 7.000 & 21 & $68,0 \pm 9,3$ & \\
\hline Campamento IV & $\sim 7.500$ & 4 & $60,5 \pm 13,5$ & \\
\hline Cima del Gasherbrum II & 8.035 & 2 & 84 y $88 \%$ & \\
\hline
\end{tabular}

medida en el campamento base al día siguiente de llegar a él y la medida un mes después se realizó mediante la «t» de Student para muestras emparejadas. La diferencia se consideró significativa si $\mathrm{p}<0,05$.

\section{RESULTADOS}

Se resumen en la tabla 1.

$\mathrm{La} \mathrm{SaO}_{2}$ de los 8 expedicionarios en Paiyu fue del $92,9 \pm 1,4 \%$, la de los 8 expedicionarios en Gore II fue del $85,0 \pm 4,3 \%$ y la de los 8 expedicionarios en el campamento base al día siguiente de su llegada fue del 78,4 $\pm 9,5 \%$. Un mes después $\mathrm{su}_{\mathrm{SaO}}$ fue del 87,4 $\pm 3,0 \%$. Este aumento fue estadísticamente significativo $(\mathrm{p}=0,007)$.

La $\mathrm{SaO}_{2}$ de 6 sujetos en el campamento II fue del $72,2 \pm 6,7 \% ; 10$ días después la de 4 sujetos fue del $75,5 \pm 1,7 \%$.

$\mathrm{La} \mathrm{SaO}_{2}$ de 21 sujetos en el campamento III, la tarde previa a sus respectivos intentos a cima, fue del $68,0 \pm 9,3 \%$

No fue posible recuperar el registro Holter de la $\mathrm{SaO}_{2}$ durante el sueño a $7.000 \mathrm{~m}$. Sin embargo, la información no se perdió del todo, pues el sujeto se despertó en dos ocasiones y en ambas observó en la pantalla del pulsioxímetro una $\mathrm{SaO}_{2}$ del $40 \%$.

$\mathrm{La} \mathrm{SaO}_{2}$ en el campamento IV fue del 60,5 \pm $13,5 \%$. Las cifras de los 4 sujetos fueron 78, 64, 52 y $48 \%$ respectivamente.

$\mathrm{La} \mathrm{SaO}_{2}$ de dos sujetos en la cima del Gasherbrum II fue del 84 y del $88 \%$ respectivamente.

\section{DISCUSIÓN}

$\mathrm{La} \mathrm{SaO}$ que tuvieron los expedicionarios en Paiyu (3.365 m) fue del 92,9\%, superior a la que, según nuestras propias ecuaciones de predicción ${ }^{1}$, tendrían a la misma altitud en las montañas europeas los montañeros no aclimatados (87\%), pero es bastante similar a la que cabría esperar en personas que vivieran habitualmente a esta altitud $(94 \%)$. Ello podría explicarse por una parcial aclimatación.

El aumento significativo de la $\mathrm{SaO}_{2}$ que experimentaron los expedicionarios durante su estancia en el campamento base concuerda con el hecho de que la $\mathrm{SaO}_{2}$ aumenta durante el proceso de aclimatación, fenómeno que hemos podido poner también de manifiesto en otras montañas ${ }^{1}$. También el aumento de la $\mathrm{SaO}_{2}$ en el campamento II se interpreta como efecto de la aclimatación en curso. Debe tenerse presente, no obstante, que las cifras correspondientes al campamento II no son comparables porque no se obtuvieron sobre los mismos sujetos.

Nuestra serie de 21 personas estudiadas a $7.000 \mathrm{~m}$ es probablemente la mayor serie publicada en el mundo de sujetos cuya $\mathrm{SaO}_{2}$ se ha medido a esa altitud. La cifra del $68 \%$ es muy similar a la que hace 18 años habíamos calculado teóricamente para esta altitud ${ }^{4}$

Esta información nos permite calcular aproximadamente qué contenido total de oxígeno en sangre arterial tienen los montañeros sanos a $7.000 \mathrm{~m}$ de altitud. Si suponemos para los varones una hemoglobina de $174 \mathrm{~g} / 1^{5}$, una saturación arterial de oxígeno del $68 \%$ y una presión parcial de oxígeno en sangre arterial de $32 \mathrm{mmHg}^{6}$, el contenido total de oxígeno en sangre arterial oscila en torno a $159,5 \mathrm{ml}$ por litro de sangre. Al nivel del mar se suele considerar normal entre 180 y $210 \mathrm{ml}$ de oxígeno por litro de sangre. En esas condiciones basta un incremento relativamente pequeño del gasto cardiaco para mantener el transporte de oxígeno a un nivel similar al que tienen los sujetos sanos al nivel del mar.

La cifra de $\mathrm{SaO}_{2}$ del $40 \%$ medida en un sujeto en dos ocasiones durante la noche es excepcionalmente baja. El sujeto (JBM) es un médico intensivista acostumbrado a usar pulsioxímetros, y en ambos casos comprobó que la pinza del pulsioxímetro estaba bien colocada, la mano estaba caliente (la medición tenía lugar dentro del saco de dormir) y la pantalla daba buena señal. El sujeto se encontraba completamente lúcido y sin disnea. Esta reducción de la $\mathrm{SaO}_{2}$ durante la noche pudo tener relación con la hipoventilación que se produce habitualmente durante el sueño, especialmente a gran altitud ${ }^{7,8}$.

Las cifras de $\mathrm{SaO}_{2}$ medidas en la cima del Gasherbrum II son ciertamente sorprendentes, y hay que aceptarlas con cautela porque, a primera vista, parece que vayan en contra del sentido común. En primer lugar, surge la duda de si son fiables o no. Están obtenidas sólo sobre dos sujetos, pero la persona que hizo la medición (RRS) es médico, está acostumbrada al uso de pulsioxímetros en la montaña y no recuerda haber tenido dificultades para hacer la lectura. A priori estas cifras, por increíbles que nos parezcan, no tienen por qué ser menos fiables que otros datos obtenidos a gran altitud que, en su día, parecieron igualmente inverosímiles (por ejemplo, la $\mathrm{PaCO}_{2}$ de 7,5 $\mathrm{mmHg}$ en las muestras de aire alveolar obtenidas por 
Chris Pizzo en la cumbre del Everest ${ }^{9}$ ). En segundo lugar, si estas cifras son ciertas, parece difícil explicar el hecho de que $\mathrm{SaO}_{2}$ a $8.035 \mathrm{~m}$ sea mayor que a 7.000 $\mathrm{m}$. Sin embargo, este hecho coincide con los resultados de un estudio teórico ${ }^{6}$ en el que uno de nosotros calculó (antes de que se generalizase el uso del pulsioxímetro portátil) cómo disminuye la $\mathrm{SaO}_{2}$ con la altitud a partir de los datos publicados por diversos autores, entre ellos West et al en la American Medical Research Expedition to Everest ${ }^{9-11}$. Según el mencionado estudio, si los datos de West et al son ciertos, la $\mathrm{SaO}_{2}$ disminuiría progresivamente con la altitud hasta llegar a un valor del $65 \%$ a $7.500 \mathrm{~m}$, pero por encima de esta cota aumentaría con la altitud. La causa de este aumento de la $\mathrm{SaO}_{2}$ sería la hiperventilación, que por una parte aumenta la presión parcial de oxígeno en el aire alveolar $\left(\mathrm{PaO}_{2}\right)$ y por la otra produce alcalosis respiratoria, lo que desplaza hacia la izquierda la curva de saturación de la hemoglobina.

En cambio, este comportamiento de la $\mathrm{SaO}_{2}$ por encima de $7.500 \mathrm{~m}$ no coincide con las cifras de $\mathrm{SaO}_{2}$ en reposo (considerablemente más bajas) que se midieron en los voluntarios que participaron en las operaciones Everest II $^{12}$ y Everest III $^{13}$. Una explicación posible es que nuestros sujetos, que acababan de ascender a la cima del Gasherbrum II por su propio esfuerzo, podían estar hiperventilando más que los voluntarios de aquellas operaciones, que habían sido sometidos pasivamente a la despresurización en sus respectivas cámaras hipobáricas. De hecho, la hiperventilación es una característica del montañismo en altitudes extremas. Hiperventilar en altitud cuesta menos esfuerzo que al nivel del mar debido a que la viscosidad del aire es mucho menor. El efecto de la hiperventilación sobre la $\mathrm{SaO}_{2}$ es perceptible incluso en altitudes no extremas: Avellanas et al comprobaron en varias cumbres de los Pirineos que la hiperventilación voluntaria mejoraba la $\mathrm{SaO}_{2}$ hasta igualar las cifras que los mismos sujetos tenían en Huesca antes de la ascensión ${ }^{14}$.

La principal objeción que puede hacerse a nuestro estudio es la relativa a la exactitud de nuestras mediciones. Antes de emprender nuestros estudios en altitud realizamos un test de Altmann y comprobamos que existía una correlación aceptable entre la $\mathrm{SaO}_{2}$ medida por pulsioximetría y la medida por gasometría arterial en 150 pacientes estudiados en el Laboratorio de Pruebas Funcionales Respiratorias del Hospital La Fe ${ }^{1}$. Torre Bouscoulet et al ${ }^{15}$ comprobaron la precisión y exactitud de un pulsioxímetro de bolsillo en 96 pacientes a los que se hizo gasometría arterial a $2.240 \mathrm{~m}$ de altitud y hallaron un error de sólo el $0,28 \pm 3,1 \%$. En un estudio de Benoît et al ${ }^{16}$ la diferencia entre la $\mathrm{SaO}_{2}$ determinada por pulsioximetría y la medida por gasometría arterial fue sólo del $1,8 \%$ para una $\mathrm{SaO}_{2}$ del $57-75 \%$. También se suele argüir que las mediciones en altitud pueden estar artefactadas por el frío. Sin embargo, dos de los autores pasamos una tarde en una cámara frigorífica industrial a $-24^{\circ} \mathrm{C}$ y comprobamos que el pulsioxímetro funciona correctamente a esa temperatura y que la posible vasoconstricción cutánea no impide la medi- ción si ésta se lleva a cabo inmediatamente tras la retirada de los guantes ${ }^{1}$.

Pese a que son muchas las expediciones que disponen de pulsioxímetro, apenas se han publicado trabajos en los que se haya medido sistemáticamente la $\mathrm{SaO}_{2}$ de los alpinistas durante la ascensión a montañas de más de 8.000 metros. Los dos estudios comparables al nuestro son el británico de Peacock y Jones en el Everest ${ }^{17}$ y el alemán de Tannheimer et al en el Broad Peak ${ }^{18}$. En el primero de ellos se obtuvieron cifras de $\mathrm{SaO}_{2}$ siempre mayores que las que obtuvimos nosotros a altitudes similares, y también claramente superiores a las obtenidas en la Operación Everest II. En el segundo, sin embargo, se obtuvieron cifras de $\mathrm{SaO}_{2}$ más parecidas a las nuestras y a las obtenidas en la Operación Everest II. Ambos estudios coinciden con el nuestro en que la $\mathrm{SaO}_{2}$ de los montañeros mejoró significativamente durante la estancia en el campamento base por efecto de la aclimatación. Asimismo, en ambos se comprobó un descenso considerable de la $\mathrm{SaO}_{2}$ durante el sueño, si bien en ninguno de ellos llegó a registrarse una cifra próxima al $40 \%$ que encontramos a $\sim 7.000 \mathrm{~m}$, probablemente porque no se realizó ninguna determinación a tanta altitud. En ninguno de los dos estudios pudo registrarse la $\mathrm{SaO}_{2}$ en la cumbre; a la del Everest llegó sólo un miembro de la expedición británica y a la del Broad Peak no llegó ninguno de la alemana.

En definitiva, nuestro estudio nos ha permitido conocer cuáles son las cifras reales de $\mathrm{SaO}_{2}$ que tienen los montañeros que participan en expediciones a montañas de más de 8.000 metros. En general éstas son muy bajas, comparables a las que tienen al nivel del mar los pacientes con insuficiencia respiratoria. Las cifras de $\mathrm{SaO}_{2}$ registradas en la cima del Gasherbrum II son, por comparación, sorprendentemente altas, y es posible que se deban a la hiperventilación. No obstante, creemos que no deben ser tomadas como verdaderas hasta que no sean verificadas en posteriores estudios.

\section{Declaración de conflicto de intereses}

Los autores han declarado no tener ningún conflicto de intereses.

\section{BIBLIOGRAFÍA}

1. Botella de Maglia J, Compte Torrero L. Saturación arterial de oxígeno a gran altitud. Estudio en montañeros no aclimatados y en habitantes de alta montaña. Med Clin (Barc). 2005;124:172-6.

2. Botella J, Real R, directores. Gasherbrum II. Expedición Cinc Segles de la Universitat de València. Valencia: Universitat de València; 2001.

3. Compte Torrero L, Real Soriano R, Botella de Maglia J, de Diego Damiá A, Macián Gisbert V, Perpiñá Tordera M. Cambios respiratorios durante la ascensión a una montaña de más de 8.000 metros. Med Clin (Barc). 2002;118:47-52.

4. Botella de Maglia J. Intercambio gaseoso en la cumbre del Aconcagua. Rev Esp Cardiol. 1989;42:107-8

5. Botella de Maglia J, Martínez-Costa R. Hemorragias retinianas en las expediciones a montañas de más de 8.000 metros. Estudio de diez casos. Med Clin (Barc). 1998;110:457-61

6. Botella de Maglia J. Variación del contenido total de oxígeno en la sangre debido a la altura. En: Rañé A, Mateu M, Battestini 
R, Ricart A, Herrero R, Castelló A, directores. Medicina de montaña. Patología ambiental de la alta cota. Barcelona: Federación Española de Montañismo; 1989. p. 254-62.

7. Garrido Marín E, Botella de Maglia J. El mal de montaña. Med Clin (Barc). 1998;110:462-8.

8. Botella de Maglia J. Mal de altura. Prevención y tratamiento. Madrid: Desnivel; 2002

9. West JB, Hackett PH, Maret KH, Milledge JS, Peters RM, Pizzo CJ, et al. Pulmonary gas exchange on the summit of Mount Everest. J Appl Physiol Respirat Environ Exercise Physiol. 1983; 55:678-87.

10. West JB. Human physiology at extreme altitudes on Mount Everest. Science. 1984;223:784-8.

11. West JB. Everest the testing place. Nueva York: Mc Graw Hill; 1985.

12. Wagner PD, Sutton JR, Reeves JT, Cymerman A, Groves BM, Malconian MK. Operation Everest II: pulmonary gas exchange during a simulated ascent of Mt. Everest. J Appl Physiol. 1987; 63:2348-59.

13. Richalet JP, Robach P, Jarrot S, Schneider JC, Mason NP, Cauchy E, et al. Operation Everest III (Comex '97): effects of pro- longed and progressive hypoxia on humans during a simulated ascent to $8,848 \mathrm{~m}$ in a hypobaric chamber. En: Roach RC, Wagner PD, Hackett PH, directores. Hypoxia: into the next millennium. Nueva York: Kluwer Academic/Plenum; 1999;297-317.

14. Avellanas ML, Borderías L, Rubio S, Santolaria S, Oliván $\mathrm{M}$, Cuchí JA. Modificaciones de la saturación de oxígeno $\left(\mathrm{SO}_{2}\right)$ durante la exposición subaguda a altitud moderada. An Med Socorr Mont. 2005;2:13-9.

15. Torre Bouscoulet L, Chávez Plascencia E, Vázquez García JC, Pérez Padilla R. Precisión y exactitud de un pulsioxímetro de bolsillo en la Ciudad de Méjico. Rev Invest Clin. 2006;58:28-33.

16. Benoît H, Costes F, Feasson L, Lacour JR, Roche F, Denis $\mathrm{C}$, et al. Accuracy of pulse oximetry during intense exercise under severe hypoxic conditions. Eur J Appl Physiol Occup Physiol. 1997;76:260-3

17. Peacock AJ, Jones PL. Gas exchange at extreme altitude: results from the British 40th Anniversary Everest Expedition. Eur Respir J. 1997;10:1439-44.

18. Tannheimer $M$, Thomas $A$, Gerngroß $H$. Oxygen saturation course and altitude symptomatology during an expedition to Broad Peak (8,047 m). Int J Sports Med. 2002;23:329-35. 